\title{
WMGHMDA: a novel weighted meta-graph-based model for predicting human microbe-disease association on heterogeneous information network
}

\author{
Yahui Long and Jiawei Luo* (D)
}

\begin{abstract}
Background: An increasing number of biological and clinical evidences have indicated that the microorganisms significantly get involved in the pathological mechanism of extensive varieties of complex human diseases. Inferring potential related microbes for diseases can not only promote disease prevention, diagnosis and treatment, but also provide valuable information for drug development. Considering that experimental methods are expensive and time-consuming, developing computational methods is an alternative choice. However, most of existing methods are biased towards well-characterized diseases and microbes. Furthermore, existing computational methods are limited in predicting potential microbes for new diseases.

Results: Here, we developed a novel computational model to predict potential human microbe-disease associations (MDAs) based on Weighted Meta-Graph (WMGHMDA). We first constructed a heterogeneous information network (HIN) by combining the integrated microbe similarity network, the integrated disease similarity network and the known microbe-disease bipartite network. And then, we implemented iteratively pre-designed Weighted Meta-Graph search algorithm on the HIN to uncover possible microbe-disease pairs by cumulating the contribution values of weighted meta-graphs to the pairs as their probability scores. Depending on contribution potential, we described the contribution degree of different types of meta-graphs to a microbe-disease pair with bias rating. Meta-graph with higher bias rating will be assigned greater weight value when calculating probability scores.

Conclusions: The experimental results showed that WMGHMDA outperformed some state-of-the-art methods with average AUCs of $0.9288,0.9068 \pm 0.0031$ in global leave-one-out cross validation (LOOCV) and 5 -fold cross validation (5-fold CV), respectively. In the case studies, 9, 19, 37 and 10, 20, 45 out of top-10, 20, 50 candidate microbes were manually verified by previous reports for asthma and inflammatory bowel disease (IBD), respectively. Furthermore, three common human diseases (Crohn's disease, Liver cirrhosis, Type 1 diabetes) were adopted to demonstrate that WMGHMDA could be efficiently applied to make predictions for new diseases. In summary, WMGHMDA has a high potential in predicting microbe-disease associations.
\end{abstract}

Keywords: Microbe, Disease, Association prediction, Weighted meta-graph, Heterogeneous information network

${ }^{*}$ Correspondence: luojiawei@hnu.edu.cn

College of Computer Science and Electronic Engineering, Hunan University,

410082 Changsha, China

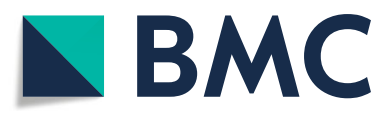

(c) The Author(s). 2019 Open Access This article is distributed under the terms of the Creative Commons Attribution 4.0 International License (http://creativecommons.org/licenses/by/4.0/), which permits unrestricted use, distribution, and reproduction in any medium, provided you give appropriate credit to the original author(s) and the source, provide a link to the Creative Commons license, and indicate if changes were made. The Creative Commons Public Domain Dedication waiver (http://creativecommons.org/publicdomain/zero/1.0/) applies to the data made available in this article, unless otherwise stated. 


\section{Background}

Accumulating clinic evidences have shown that the microbes residing in human hosts play a crucial role in the pathological mechanism of an extensive variety of human diseases. The microorganisms reside in and on human body with a wide range of organs like lung, skin, oral cavity and gut, most of which reside in gastrointestinal tract [1]. A plenty of experimental results have shown that most of commensal microbial communities benefit human health, and are even indispensable for human physiology because they could not only offer protection from pathogens and promote metablic capability, but also assist modulation of gastrointestinal development [2]. It is reported that there exist about $10^{14}$ microorganism cells inhabiting an adult intestine, which is approximately 10 times more than human cells [3]. These cells could produce a large amount of gene product which is essential for various metabolic and biochemical activities $[4,5]$. Therefore, human microbes are also often treated as a forgotten organ of human due to its similar metabolic capacity to the liver [6]. Previous studies discovered that the microbial communities were significantly affected by the genetics [7-9] as well as the dynamic habitat environments, such as season [10], smoking [11], diets [12] and antibiotics [13]. The dynamic changes of these factors can lead to the imbalance of microbial communities and further affect the biological progress (i.e., metabolism, proteomic) of associated microbes, which possibly motivates a variety of important human diseases, such as asthma [14], diabetes [15], liver diseases [16], and even cancer [17].

Since the first microorganism which can cause human disease was found in the 1800 s, an increasing number of microorganisms have been demonstrated to be the causation of different human diseases. For example, in order to determine the relationship between the clinical features of asthma and the composition of the airway bacterial microbiota, Huang et al. [18] utilized culture-independent tools to detect the relative abundance and presence of most known bacteria. As a result, they demonstrated that there existed closely relevant associations between the relative abundance of members of the Comamonadaceae, Sphingomonadaceae, Oxalobacteraceae and the degree of bronchial hyperresponsiveness. Larsen et al. [19] studied the differences between the composition of the intestinal microbiota in humans with type 2 diabetes and nondiabetic persons as control and found the compositional changes of intestinal microbiota like Firmicutes, Lactobaillus, Bacteroidetes, Bacilli and Proteobacteria. Moore et al. [20] analyzed the fecal floras from five diverse polyp patients including North American Causasians, JapaneseHawaiians, rural native Africans and rural native Japanese. They eventually found the positive associations between increased risk of colon cancer and Bacteroides species and Bifidobacterium species while the closest associations of some Lactobacillus species and Eubacterium aerofacients with low risk of colon cancer. Identifying candidate microbes for diseases could not only offer insight into the pathological mechanism of human diseases, but also promote disease prevention, diagnosis, treatment and prognosis [21]. Even though the roles microbes play in the mechanism of human diseases have been increasingly revealed, a comprehensive understanding of microorganism remains largely challenge.

Considering that traditional experimental methods which researchers used to heavily depending on are time-consuming, expensive and laborious, researchers paid more attention to the development of computational methods for exploring microbe-disease associations (MDAs). A number of existing methods are implemented based on HIN consisting of multiple biological networks, such as KATAHMDA [22], RWRHMDA [23], NTSHMDA [24], PBHMDA [25]. For example, Chen et al. [22] proposed the first computational model of KATZHMDA to infer latent MDAs on HIN. In this model, all microbedisease relationship pairs are prioritized according to their probability scores obtained by calculating the numbers of walks with different lengths between microbe nodes and disease nodes. However, it is possible for this model to cause bias towards well-investigated diseases and microbes. Shen et al.[23] implemented random walk with restart (RWR) on the HIN to prioritize candidate microbe for diseases. Unfortunately, since ignoring the bias rating of tendency to be associated with different neighbor microbe nodes for disease nodes, this method fails to achieve desired prediction performance. To overcome this challenge, following it, Luo et al.[24] proposed another model NTSHMDA, which utilizes extended RWR optimized by introducing network topological similarity to rank candidate microbes for diseases. Nevertheless, the aforementioned methods only consider Gaussian kernel interaction profile similarity to calculate similarity for both disease and microbe, yet ignore rich prior information on diseases and microbes. To take advantage of such information, researchers have recently paid more attention on prioritizing disease-associated microbes by incorporating prior biological knowledge [26, 27]. As an instance, Huang et al. [26] released a microbe prioritization method, which combines two single prediction models, namely neighbor-based collaborative filtering and graph-based scoring method. This method introduces symptom-based disease similarity to improve the completeness of disease similarity. Zhang et al. [27] presented a label propagation-based method of BDSILP for ranking candidate microbes for diseases, which incorporates multiple similarities for diseases and microbes, such as disease semantic similarity and microbe functional similarity. However, for the aforementioned methods, although integrating external biological information about diseases and 
microbes into prediction model, they still fail to make predictions for new disease without any known associations.

Recently, machine learning has been applied in the bioinformatics and computational biology, such as miRNA-target association prediction [28], IncRNAdisease association prediction $[29,30]$, drug combination prediction [31], miRNA-disease association prediction [32-34] and miRNA regulatory module identification [35]. A large number of machine learning-based algorithms have been also proposed for inferring MDAs [36-38]. For example, Wang et al. [36] developed a semisupervised computational model of LRLSHMDA, which uses Laplacian regularized least squares classifier to prioritize disease-related microbes.

In this work, we proposed a novel computational model of WMGHMDA for inferring candidate microbes for diseases on HIN based on Weighted Meta-Graph. This model incorporates multiple sources of prior biological knowledge and could be applied to make predictions for new diseases without any known associations. Our approach involves three steps. First, a HIN is constructed by connecting the integrated microbe and disease similarity networks via observed microbe-disease bipartite network. Next, a pre-designed Weighted Meta-Graph search algorithm is iteratively implemented on the HIN to enumerate weighted meta-graphs related to each microbedisease pair. Finally, we calculate the probability score for each microbe-disease pair by summing up the contribution values of relevant weighted meta-graphs and prioritize candidate microbes for diseases according to their probability scores. We carried out comprehensive experiments to evaluate the prediction performance of our method and demonstrated the improvement of prediction accuracy compared to state-of-the-art methods. In particular, WMGHMDA is capable of recovering average $75.4 \%$ of known true positive samples in the top-100 prediction for three complex human diseases.

Mainly, our contributions are as follows:

(1) We propose a novel computational model of WMGHMDA for predicting MDAs, which is the new application of meta-graph. To our knowledge, WMGHMDA is the first tool to use weighted meta-graph for microbe-disease association prediction.

(2) To improve the completeness of similarity, multiple prior biological knowledge is introduced in this paper, including disease semantic similarity and microbe functional similarity, which effectively boosts the improvement of prediction accuracy.

(3) In the network, subtle semantics between diseases and microbes are prolifically hidden. To capture this feature, we generalize common unweighted meta-graph to weighted meta-graph based on which we design a novel Weighted Meta-Graph search algorithm and leverage it to prioritize candidate microbes for diseases.

(4) Comparisons with state-of-the-art methods on HMDAD demonstrate the superiority of our approach. In addition, the approach is particular effective for a new disease with few known related microbes or without any known related microbes.

\section{Related work}

Recently, a large number of tools have been developed for identifying MDAs. Most of existing methods are based on the assumption proposed by Ma et al. [21] that the functionally similar microbes tend to present interaction or non-interaction with phenotypically similar diseases and vice versa.

Predicting MDAs based on network analysis has become popular $[22,25,27,39,40]$. These methods attempt to infer the possibility of existing associations between diseases and microbes through HIN consisting of different biological networks. For example, Chen et al. [22] proposed a computational model of KATZHMDA based on HIN. This model infers potential association pairs using KATZ measurement on the network. Huang et al. [25] leveraged a special depth-first search framework on HIN for predicting candidate microbes for diseases. However, such methods calculate the similarities for both diseases and microbes strongly depending on Gaussian kernel similarity, which, as a result, tends to "recommend" well-studies microbes with more known associated diseases. In contrast, our proposed method combines multiple prior knowledge and alleviates this problem.

Random walk has been applied for prioritizing candidate microbes for diseases [23, 24, 41, 42]. Most of these methods are developed based on RWR, the variant of random walk. They make full use of the advantage of RWR in capturing local and global network topological characteristics. As an instance, Shen et al. [23] utilized extended random walk to uncover disease-related microbes but failed to consider the bias rating for different association pairs. To tackle such problem, Luo et al. [24] further improved this model by introducing network topological similarity. Unfortunately, these methods are limited in inferring possible microbes for diseases with few known associated microbes or without any known associated microbes. In this paper, our method is able to make predictions for new diseases by applying weighted meta-graph that could identify hidden subtle semantic relations between a disease and considered microbes only if the similarity feature can be obtained for the disease.

More and more attention has been recently paid to the application of machine learning in the prediction of MDAs [36-38]. Most of these methods achieves the prediction based on matrix factorization. For example, Shen 
et al. [38] introduced collaborative matrix factorization to update the correlation matrix of diseases and microbes for ranking candidate microbes for diseases. He et al. [37] released a novel computational methods of GRNMFHMDA based on graph regularized non-negative matrix factorization, but the selection of optimal parameters for this method remains a challenge. In addition, Wang et al. [36] proposed a semi-supervised computational model of LRLSHMDA, which uses Laplacian regularized least squares classifier to prioritize disease-related microbes.

\section{Results}

\section{Performance evaluation}

In order to measure the prediction accuracy of the proposed WMGHMDA model, we implement global LOOCV and 5-fold CV on HMDAD, respectively. In the framework of LOOCV, each observed microbe-disease pair is selected as test sample in turn while the rest observed microbe-disease association pairs are considered as training samples. In each round, the test sample is ranked according to its prediction score against all candidate samples. Here, candidate samples refer to the unverified microbe-disease association pairs. If the rank of the test sample is higher than the given threshold, the proposed method is regarded as successful in inferring the tested microbe-disease pair. Similar to LOOCV, all observed microbe-disease association pairs are randomly divided into five groups in 5-fold CV. Each group is left out in turn to test model while the remaining groups are adopted as training samples. To weak the impact of the bias resulting from the progress of random division to experimental results, this progress is executed 100 times. It is worth noting that both the similarities of microbes and diseases need to be recalculated for each round in both LOOCV and 5-fold CV. For the sake of convenient observation, we draw the receiver-operating characteristics (ROC) curves by plotting true positive rate (TPR, sensitivity) against false positive rate (FPR, 1-specificity) based on different thresholds. Sensitivity represents the percentage of the true positive samples which are ranked higher than the given threshold in the whole positive samples. Specificity means the percentage of the negative samples with ranks lower than the given threshold in the whole negative samples. Area under ROC curve (AUC) is further calculated as a metric to measure the prediction capability of WMGHMDA. If the value of AUC is equal to 1 , it means the model obtains perfect performance. If the value of AUC is equal to 0.5, it represents the performance of the model is random. As a result, WMGHMDA achieved an effective and reliable performance with average AUCs of 0.9288, $0.9068 \pm 0.0031$ in the frameworks of LOOCV and 5-fold CV, respectively, as shown in Fig. 1.

For assessing the robustness of our method, we randomly select some known associations as unknown associations. The percentage varies from 10 to $30 \%$. Global LOOCV is then implemented on the new known microbe-disease associations to evaluate the performance of our method. The results have been shown in Table 1, from which we can find that the AUCs are stable with the percentage increasing. It indicates that our method is slightly limited to the effect of sparse evidences.

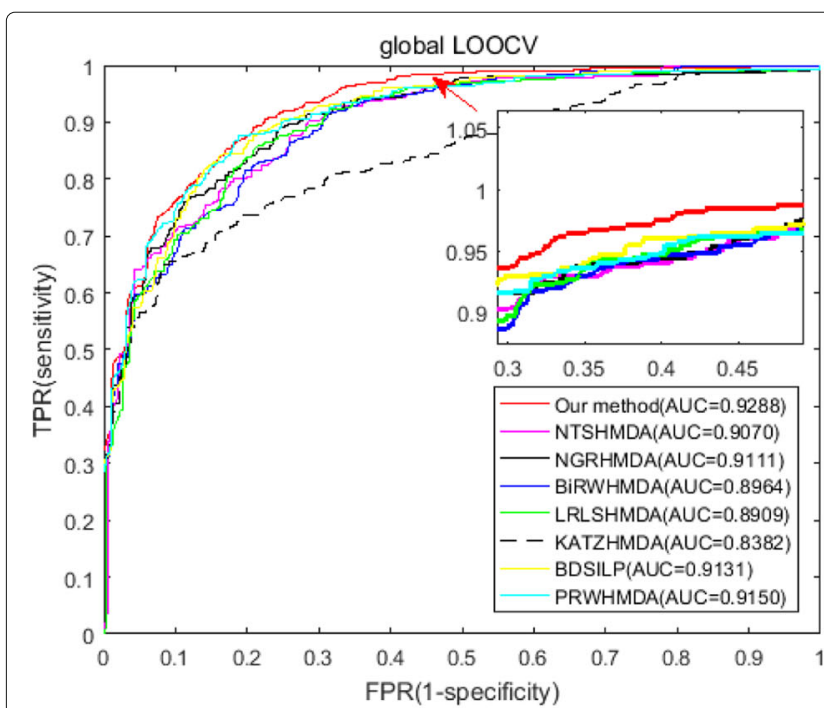

(a)

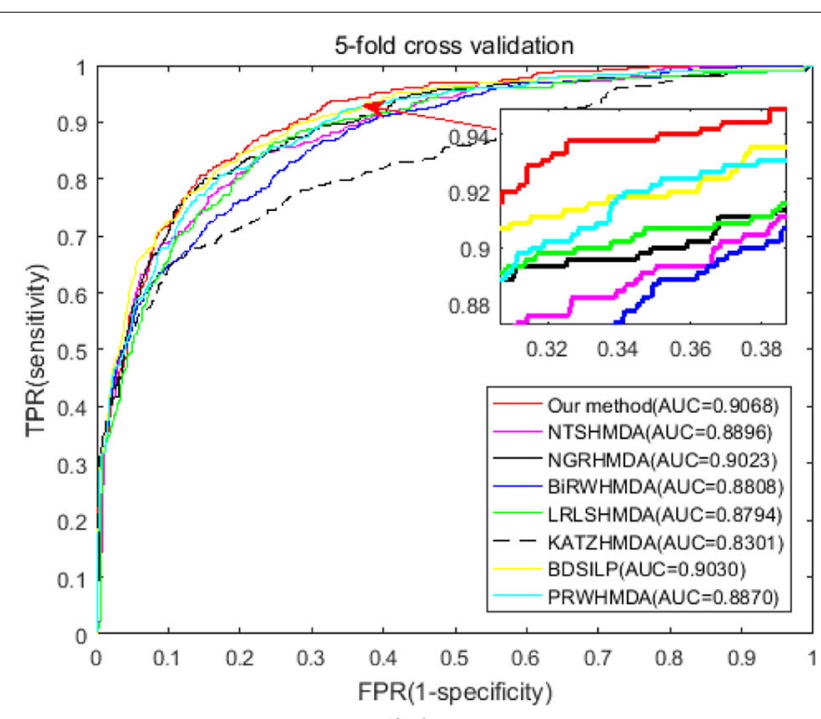

(b)

Fig. 1 Comparisons of prediction performance between our method and five state-of-art prediction models (NTSHMDA, NGRHMDA, BiRWHMDA, LRLSHMDA, KATZHMDA, BDSILP and PRWHMDA) in the frameworks of global LOOCV and 5-fold CV, respectively. a The ROC curves and AUC values based on LOOCV, b The ROC curves and AUC values based on 5-fold CV 
Table 1 The robustness of WMGHMDA

\begin{tabular}{llll}
\hline Percentage & $10 \%$ & $20 \%$ & $30 \%$ \\
\hline AUC & 0.9222 & 0.9161 & 0.9050 \\
\hline
\end{tabular}

\section{Comparison with other methods Do novo cross-validation}

In order to further validate the sound prediction performance of the model, we compare WMGHMDA with some state-of-the-art computation methods, such as KATZHMDA [22], NTSHMDA [24], NGRHMDA [26], BiRWHMDA [41], LRLSHMDA [36], BDSILP [27] and PRWHMDA [42]. KATZHMDA is the first computation model to infer latent MDAs. It prioritizes candidate microbe-disease pairs according to their prediction scores obtained by calculating the numbers of walks with different lengths between microbe nodes and disease nodes. NTSHMDA is a global computational model that infers potential MDAs using optimized random walk with restart by introducing network topological similarity. NGRHMDA integrates two single recommendation algorithms, namely neighbor-based prediction model and graph-based prediction model, to calculate relationship probabilities of microbe-disease pairs and further prioritizes potential candidate microbes for diseases according to their probabilities. BiRWHMDA achieves possible microbe-disease association inference by exploring the CBGs through iteratively implementing random walk on the disease similarity networks and the microbe similarity network. LRLSHMDA is a semi-supervised computation model that uncovers potential MDAs by introducing Laplacian regularized least squares classifier. BDSILP is a network-based microbe prioritization model using label propagation. PRWHMDA finishes the inference of microbe-disease associations with extended RWR optimized by Particle Swarm Optimization. All of these methods perform great prediction performance. In the sake of fair comparison, these contrast approaches are implemented on the same database HMDAD, including 483 entries between 39 diseases and 292 microbes, as WMGHMDA. Both global LOOCV and 5-fold CV are adopted to measure the inference capabilities of experimental methods. As shown in Fig. 1, WMGHMDA outperforms baseline methods with an AUC of 0.9288 in LOOCV while the AUCs of KATZHMDA, NTSHMDA, NGRHMDA, BiRWHMDA, LRLSHMDA, BDSILP and PRWHMDA are 0.8382, 0.9070, 0.9111, 0.8964, 0.8909, 0.9131 and 0.9150 , respectively. Similarly, in 5-fold CV, the performance of WMGHMDA is also superior to baseline methods (KATAHMDA 0.8301 \pm 0.0033 ; NTSHMDA $0.8896 \pm 0.0038$; NGRHMDA $0.9023 \pm 0.0031$; BiRWHMDA $\quad 0.8808 \pm 0.0029 ; \quad$ LRLSHMDA $\quad 0.8794 \pm 0.0033$, BDSILP $0.9030 \pm 0.0039 ;$ PRWHDMA $0.8870 \pm 0.0046$ ) with average AUC of $0.9068 \pm 0.0031$.
Furthermore, according to the result of global LOOCV, we obtain precision, recall and F1-score (See Additional file 1: Table S1) with different threshold $k$. We give the results of different methods with threshold $k$ varying from 0 to 50 with a step value of 5 . It can be clearly observed in Additional file 1: Table S1 that from the top-1 to -10 predictions, our model outperforms baseline methods in terms of these three evaluation metrics. For the predictions from top-10 to -50 , our approach is comparable or even superior to baseline methods. It indicates that our method is effective in identifying candidate microbe for diseases. In addition, we can notice that the performances of some baseline methods (i.e., BiRWHMDA, NGRHMDA, KATZHMDA) are close to that of our approach. It could be explained that the difference of the prediction ability is possibly weakened by the highly skewed dataset where the number of unknown associations greatly exceeds the number of known associations in our database [43]. We believe that with the validation of more known evidences, the difference will become more evident.

\section{Evaluate the performance of WMGHMDA in recovering known associations}

In order to compare the ability of different methods in recovering a true association, we give the cumulative distribution of known associations recovered with top 10, 50, 100, 150 and 200 predictions, as shown in Fig. 2. Also, this result is obtained based on the result of global LOOCV. We can see in Fig. 2 that the number of known associations truly recovered by our method is more than those of baseline methods for all thresholds while our method is slightly inferior to NTSHMDA in top-100 prediction. Therefore, we can conclude that the developed model of WMGHMDA is effective and reliable, and, moreover, has comprehensively higher accuracy in inferring potential candidate microbes for diseases than state-of-the-art algorithms.

\section{Performance of prediction for new diseases}

An important aspect to evaluate a novel model is the ability to make predictions for new diseases. New diseases refer to the diseases which have no any known experimentally verified associated microbes, but have prior features available for prediction. Due to the lack of known microbe associations, few existing computational methods are capable of inferring potential microbes for new diseases in the research field of MDAs. Therefore, one of our main contributions is that the proposed method is able to predict candidate microbes for new disease, which is achieved by taking prior information related to this disease and specific microbes into account. For the purpose of evaluation, we select the cumulative distribution of the ranks as a measure criterion to distinguish the 


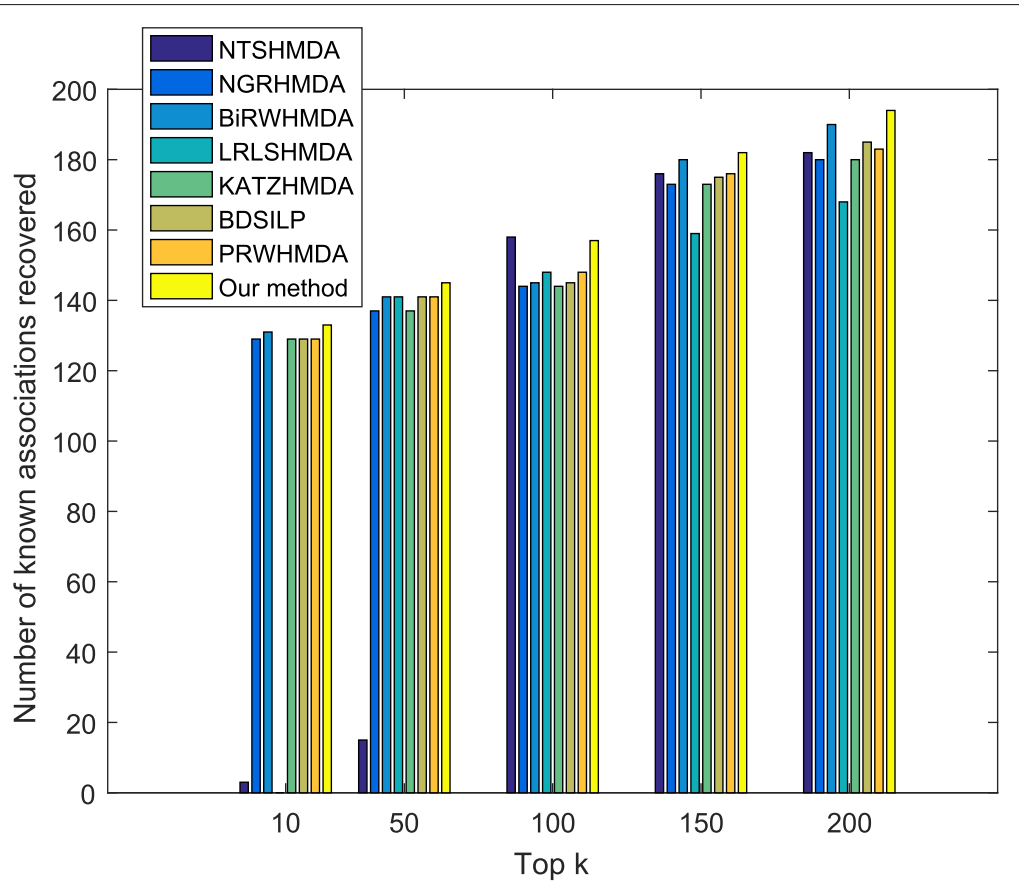

Fig. 2 Performance comparisons between our method and baseline methods (NTSHMDA, NGRHMDA, BiRWHMDA, LRLSHMDA, KATZHMDA, BDSILP and PRWHMDA) in recovering known associations

prediction performance of different models for new diseases. This measure has been adopted for evaluation in multiple research fields of computational biology [44-47]. Considering that most of diseases included in HMDAD only have a small number of positive samples, and adopting them as test samples possibly results in the bias of evaluation, we choose three common diseases (Crohn's disease, Liver cirrhosis, Type 1 diabetes) with more positive samples as test samples for more accurate evaluation.

For each of these diseases, we first artificially set all the known associations between microbes and test disease as unknown ones. And then different models are carried out on this test set to obtain the ranks of microbes which are experimentally verified to be associated with this test disease. After that, we can plot the average cumulative distribution of the ranks for three diseases, as shown in Fig. 3 where $\mathrm{x}$-axis represents the top- $k$ predicted microbes and $y$-axis denotes the probability of recovering an observed association in the top- $k$ prediction. Note that all baseline methods are missing from the plot, as they cannot be applied for prediction for new diseases without any known associations. In Fig. 3, we can obviously observe that with the increase of number of microbes looked at, the percent of known true positive samples recovered by our method constantly increases. Especially, our model successfully recovers average $75.4 \%$ of known associations in the top-100 predictions. This performance can be explained because for any specific disease with known or unknown associations, in the framework of WMGHMDA, weighted meta-graph is capable of effectively capturing potential semantic associations between this disease and candidate microbes by combining prior biological knowledge associated with this disease and microbes. Thus, it can be concluded that our method is reliable and effective in predicting potential microbes for new diseases.

\section{The effects of parameters on WMGHMDA}

In this section, we evaluate the impacts of parameters on the performance. $\alpha$ is weighted factor defined to weight the effects of the disease semantic similarity and the Gaussian kernel disease similarity to the integrated disease similarity. We set $\alpha$ from 0.1 to 0.9 with a step value of 0.1 . $\beta$ is a weighted factor used to control the contribution of the microbe functional similarity to the integrated microbe similarity. The setting of $\beta$ is similar to $\alpha$ varying from 0.1 to 0.9 . For the convenience of parameter tuning, one parameter is tested with the remaining parameters fixed. As shown in Fig. 4a, b, it can be obviously observed that the AUC first increases, and then decreases for both $\alpha$ and $\beta$. The best performance can be obtained when $\alpha$ and $\beta$ are set as 0.6 and 0.7 , respectively. It demonstrates that the values of $\alpha$ and $\beta$ that are large or small are not good for the improvement of the prediction accuracy of our approach. $\mu$ is a weight factor controlling the contribution of weighted meta-graph to the prediction probability. Figure 4c shows that with $\mu$ increasing, the 


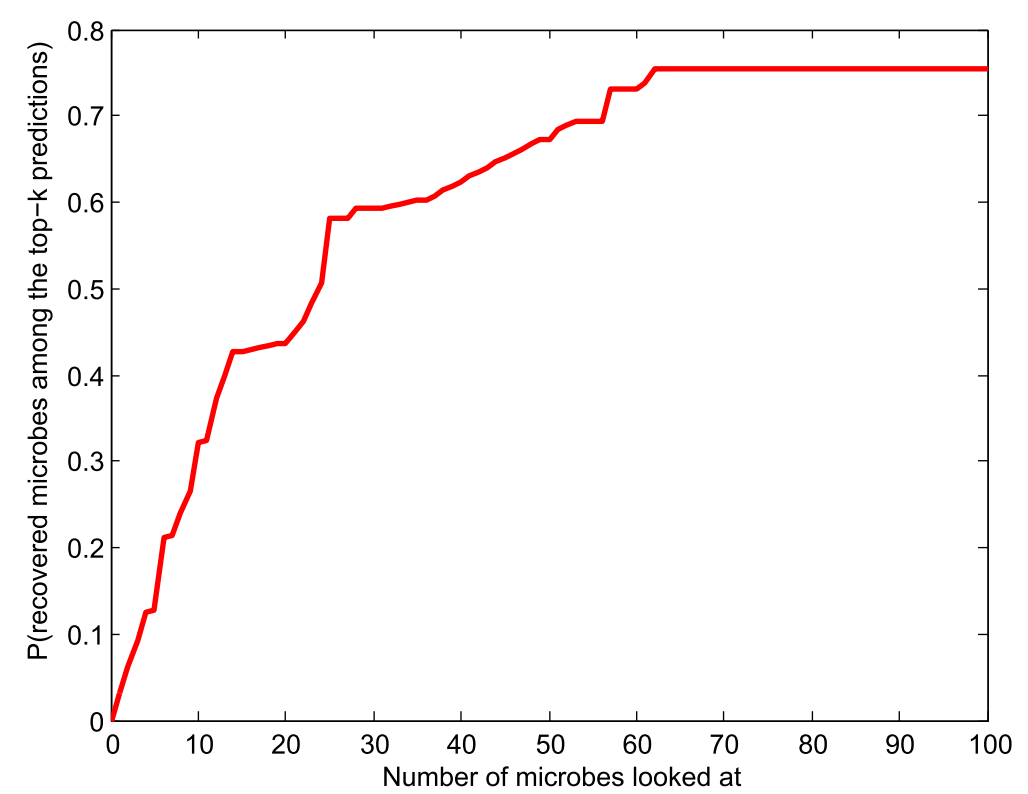

Fig. 3 Performance of WMGHMDA in inferring candidate microbes for new diseases

AUC always presents an upward trend, and we can acquire the best result when $\mu$ is set as 0.9 . The result validates the effectiveness of weighted meta-graph.

\section{Case studies on asthma and inflammatory bowel disease}

To further verify the prediction accuracy of WMGHMDA, we take asthma and Inflammatory bowel disease (IBD) as two case studies which are implemented on our model. All candidate microbes for asthma and IBD are prioritized according to their prediction scores. Here, the performance of WMGHMDA is evaluated by observing the number of confirmed candidate microbes ranked in the top of 10,20, 50 for a specific disease. It is necessary to point out that for a given disease, if one microbe is associated with this disease, the corresponding genus of this microbe is also assumed to be related with this disease.
Finally, 9, 19, 37 and 10, 20, 45 out of the top-10, 20, 50 candidate microbes could be manually validated based on previous literatures for asthma and IBD, respectively.

\section{Asthma}

Asthma is a common long-term inflammatory disease of the airway of the lungs [48]. An increasing number of statistics have shown that the microorganisms living in or on human hosts significantly get involved in the pathological mechanism of asthma. Nine out of top-10 microbes inferred to be associated with asthma obtain validation from different literatures. For example, Marri et al. [49] compared the changes of the microbiome of induced sputum from both asthmatic and nonasthmatic adults. As a result, they found that Firmicutes occurred in samples from nonasthmatic subjects with higher frequency.

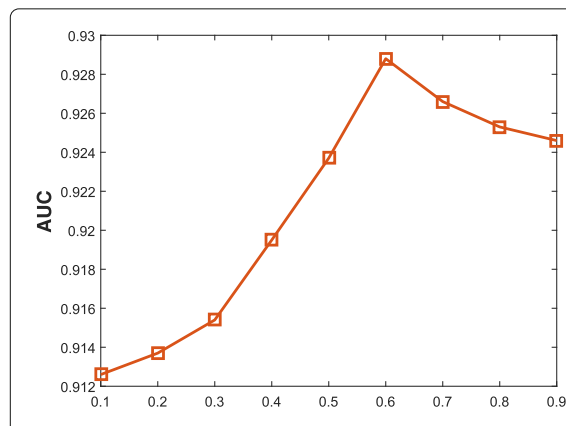

(a)

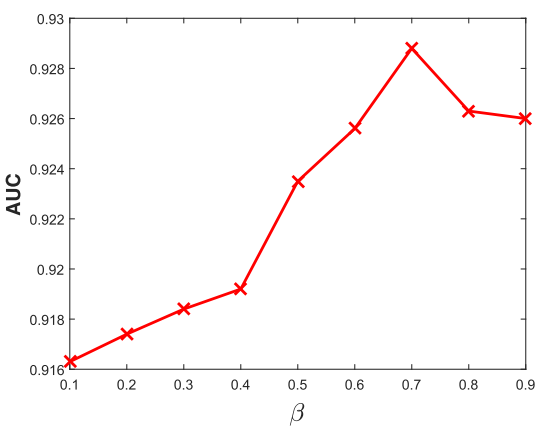

(b)

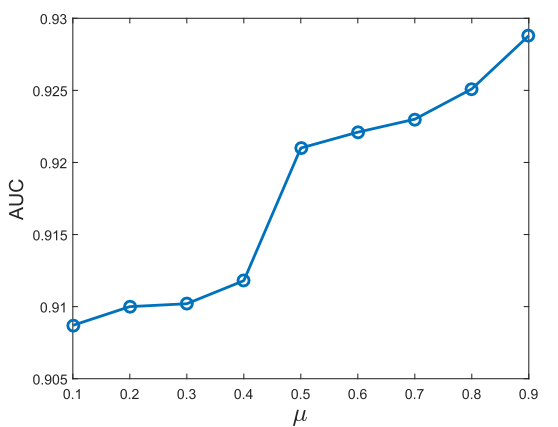

(c)

Fig. 4 The impacts of parameters on the performance. (a) $\alpha$, (b) $\beta$, (c) $\mu$ 
Lachnospiraceae was found to be significantly more prevalent in the sputum of asthma patients than in the sputum of the controls [50]. It was discovery that the abundance of Enterobacteriaceae of severe asthmatics was higher than that of non-severe asthmatics [51]. Yu et al. [52] showed that Lactobacilli were probiotic bacteria and had potential for preventing asthma. The abundances of Staphylococcus were presented to be larger in asthmatic children than those in healthy controls and asthmatics [53]. Vael et al., [54] investigated the relationship between the development of asthma and early intestinal colonization in the first 3 years of life, which, eventually, showed that the count of Bacteroides fragillis was significantly higher in children with a positive index compared to those without at 3 weeks. This result indirectly demonstrates that the change of Bacteroides is associated with asthma. Only Clostridium difficile has been not validated nowadays. Top-10 inferred candidate asthma-associated microbes are also listed in Table 2. Furthermore, 19, 37 out of top-20, 50 candidate microbes for asthma are confirmed manually by previously published literatures, as shown in Table 2.

\section{Inflammatory bowel disease}

Inflammatory bowel disease is a common group of inflammatory conditions of the colon and small intestine. Similar to asthma, WMGHMDA is also applied to infer possible related microorganisms for IBD. As a result, 10 of top10 candidate IBD-related microbes could be confirmed by current researches. As an instance, it was demonstrated that the decreases of abundances of Bacteroidete and Firmicute were associated with the formation of IBD [56]. The abundance of Clostridium coccoides was discovered to be less represent in Crohn's disease patients than healthy objects [57]. It was confirmed that there was an inversely association between the presence of Heicobacter pylori and IBD [58]. Azimirad et al. [59] indicated that there existed significant relationships between IBD and

Table 2 Prediction results of the top 50 asthma-associated microbes

\begin{tabular}{|c|c|c|c|}
\hline Microbe & Evidence & Microbe & Evidence \\
\hline Firmicutes & PMID:23265859 & Treponema & Unconfirmed \\
\hline Clostridium difficile & Unconfirmed & Porphyromonas gingivalis & PMID:20308298 \\
\hline Actinobacteria & PMID:28947029 & Selenomonas & PMID:27093794 \\
\hline Clostridium coccoides & PMID:21477358 & Escherichia coli & PMID:29161804 \\
\hline Lactobacillus & PMID:20592920 & Clostridium leptum & PMID:29445257 \\
\hline Lachnospiraceae & PMID:27433177 & Gammaproteobacteria & PMID:28947029 \\
\hline Staphylococcus & PMID:29445257 & Fusobacterium nucleatum & {$[55]$} \\
\hline Enterobacteriaceae & PMID:28947029 & Alcaligenaceae & PMID:19407055 \\
\hline Bacteroides & PMID:18822123 & Coriobacteriaceae & PMID:28947029 \\
\hline Veillonella & PMID:25329665 & Erysipelotrichaceae & Unconfirmed \\
\hline Clostridia & PMID:21477358 & Methanobrevibacter smithii & Unconfirmed \\
\hline Fusobacterium & {$[55]$} & Bacteroidaceae & PMID:28947029 \\
\hline Enterococcus & PMID:29788027 & Verrucomicrobiaceae & Unconfirmed \\
\hline Burkholderia & PMID:24451910 & Dietzia maris & Unconfirmed \\
\hline Streptococcus & PMID:17950502 & Staphylococcus epidermidis & PMID: 29569134 \\
\hline Enterobacter aerogenes & PMID:24973962 & Tropheryma whipplei & PMID:26647445 \\
\hline Enterobacter hormaechei & PMID:24973962 & Acinetobacter & PMID:29447223 \\
\hline Klebsiella pneumoniae & PMID:24958709 & Corynebacterium & PMID:29885665 \\
\hline Shigella dysenteriae & Unconfirmed & Oxalobacter formigenes & Unconfirmed \\
\hline Propionibacterium & PMID:27433177 & Desulfovibrio & Unconfirmed \\
\hline Propionibacterium acnes & PMID:27433177 & Lysobacter & Unconfirmed \\
\hline Pseudomonas & PMID:13268970 & Rickettsiales & Unconfirmed \\
\hline Stenotrophomonas maltophilia & Unconfirmed & Streptococcus & PMID:17950502 \\
\hline Faecalibacterium prausnitzii & PMID:27253486 & Xanthomonas & Unconfirmed \\
\hline Actinomyces & PMID:23326024 & Clostridium & PMID:26565810 \\
\hline
\end{tabular}

The first column records top 1-25 associated microbes. The third column records top 26-50 associated microbes 
Clostridiu difficile and staphylococcus. Through observing the composition of salivary microbiota from 35 IBD patients, it was uncovered that Haemophilus largely contributed to dysbiosis observe in the salivary microbiota from IBD patients [60]. Ten out of top-10 predicted candidate microorganisms considered to be associated with IBD are also listed in Table 3, from which we can found that only Enterobacteriaceae has not been confirmed by current researches. In addition, 20 out of top- 20 candidate, 45 out of top-50 candidate microbes for IBD are manually validated by current researches, as shown in Table 3. In addition, the network of the top-50 predicted associations for IBD and asthma obtained by our model is shown in Additional file 2: Figure S1. Obviously, it is observed that a microbe is possibly associated with one or more diseases. In a word, these two sets of case studies validate the powerful capability of our method in inferring new possible microbes for diseases again.

\section{Discussion}

Evidences showed that the microbes living in or on human body significantly contributed to the induction progress of an extensive varieties of complex human diseases, including formation, development and progression. Inferring latent candidate microbes for diseases can not only provide significant insights into the understanding of the pathological mechanism of complex diseases, but also promote disease prevention, diagnosis and treatment, as well as drug development. In this study, we proposed a novel Weighted Meta-Graph based computational method of WMGHMDA to predict potential microbe-disease associations based on HIN. The experimental results indicated that our method achieved a desired improvement compared to some state-of-the-art methods. Our method made full use of multiple prior biological knowledge.

In particular, we integrated disease semantic similarity and microbe functional similarity to complement and

Table 3 Prediction results of the top 50 inflammatory bowel disease-associated microbes

\begin{tabular}{|c|c|c|c|}
\hline Microbe & Evidence & Microbe & Evidence \\
\hline Bacteroidetes & PMID:25307765 & Clostridium & Azimirad et al.,2012 \\
\hline Firmicutes & PMID:25307765 & Bacteroides ovatus & PMID:29454108 \\
\hline Prevotella & PMID: 25307765 & Betaproteobacteria & Unconfirmed \\
\hline Clostridium difficile & Azimirad et al.,2012 & Clostridiales & PMID:29965986 \\
\hline Helicobacter pylori & PMID:22221289 & Klebsiella & PMID:29573336 \\
\hline Clostridium coccoides & PMID:19235886 & Bifidobacterium & PMID:24478468 \\
\hline Staphylococcus & Azimirad et al.,2012 & Gammaproteobacteria & PMID:29385143 \\
\hline Lactobacillus & PMID:26340825 & Porphyromonadaceae & PMID:29573237 \\
\hline Haemophilus & PMID:24013298 & Collinsella aerofaciens & PMID:26848182 \\
\hline Enterobacteriaceae & PMID:24629344 & Propionibacterium & PMID:26640113 \\
\hline Staphylococcus & Azimirad et al.,2012 & Propionibacterium acnes & PMID:26640113 \\
\hline Veillonella & PMID:28842640 & Alistipes & PMID:28877044 \\
\hline Bacteroides & PMID:25307765 & Parabacteroides & PMID:25307765 \\
\hline Clostridia & PMID:25307765 & Prevotellaceae & PMID:29514953 \\
\hline Bacteroides vulgatus & PMID:29454108 & Veillonellaceae & PMID: 28842640 \\
\hline Bacteroides uniformis & PMID:26789999 & Fusobacteriaceae & PMID:24629344 \\
\hline Bacteroidaceae & PMID:17897884 & Shigella & PMID:29485143 \\
\hline Faecalibacterium prausnitzii & PMID:24799893 & Enterobacter aerogenes & Unconfirmed \\
\hline Streptococcus & PMID:23679203 & Enterobacter hormaechei & Unconfirmed \\
\hline Clostridium leptum & PMID:28099495 & Klebsiella pneumoniae & PMID:29573336 \\
\hline Enterococcus & PMID:24629344 & Coxiellaceae & Unconfirmed \\
\hline Escherichia coli & PMID:29573336 & Bacteroidales & PMID:24629344 \\
\hline Stenotrophomonas maltophilia & Uncofirmed & Enterococcus faecium & PMID:29135456 \\
\hline Fusobacterium & PMID:25307765 & Erysipelotrichales & PMID:29965986 \\
\hline Burkholderia & PMID:24325678 & Bacilli & PMID:29049404 \\
\hline
\end{tabular}

The first column records top 1-25 associated microbes. The third column records top 26-50 associated microbes 
improve the disease similarity and microbe similarity, respectively. This prior information is essential for the predictions for new diseases. The prediction experiments for three common complex diseases indicated that prior information was helpful for making predictions for new diseases. In fact, the introduction of prior information also alleviates the problem that previous computational methods tend to "recommend" well-investigated candidate microbes or diseases. In this study, we introduced meta-graph to solve the problem of inferring potential associations between diseases and microbes with the consideration of its power in capturing complex semantics in HIN. Further, inspired by the fact that there are prolific subtle semantics hidden in HIN, we generalized unweighted meta-graph to weighted meta-graph to more accurately capture them. In addition, weighted meta-graphs with diverse patterns are likely to lead to differentiated contributions to a microbe-disease pair. Thus, to identify such differences and enhance the prediction accuracy, we further introduced bias rating to describe the distinct contributions of different weighted meta-graph. The comprehensive experimental results indicated that the introduction of weighted meta-graph can improve prediction performance.

The reliable performance of WMGHMDA results from several major factors as follows: to begin with, the observed experimentally validated human MDAs are reliable. In addition, the introduction of multiple prior biological information about diseases and microbes improves the completeness of similarity for diseases and microbes, which potentially enhances the prediction capability of our method. Last but not least, a crucial advantage of WMGHMDA is that it achieves potential MDAs inference based on weighted meta-graph. On the one hand, compared with unweighted meta-graph, weighted metagraph has stronger ability to capture potential subtle semantic associations between seed diseases and target microbes. On the other hand, for a microbe-disease pair, the bias contributions of different weighted meta-graphs to it are considered in this paper. Weighted meta-graph with higher bias rating is assigned greater weight value when probability score is calculated, which also promotes the improvement of the prediction performance.

Although the performance of WMGHMDA is desirable, several aspects are still expected to be further improved in future studies. Initially, the available of known MDAs is still not enough to insure more desirable prediction performance, which, however, could be addressed by adding manually more known microbe-disease association to database. Furthermore, it is greatly easy for the proposed model to suffer from decrease of accuracy owing to the high rate of false positive and false negative samples in the microbe-disease association database. Additionally, our method cannot be applied to make predictions for all new diseases. It is because for a new disease without any known evidences and DAG information, it fails to obtain its similarity between other diseases and it that is essential for new disease prediction. But this limitation could be overcome by incorporating more prior information or developing other effective similarity calculation method.

Finally, it is anticipated that the prediction accuracy of microbe-disease association could be improved through two aspects. On one hand, more prior biological knowledge could be introduced, such as microbe sequence similarity, disease gene-based similarity network and disease symptom similarity network. Compared with the study of disease similarity, the attention paid on the study of microbe similarity are relatively poor. It is an alternative way to adopt the combination of CRISPR-Cas9 with functional enrichment to measure microbe sequence similarity by first mapping genetic interaction network based on microbial sequencing data and then detecting similar features on the network. On the other hand, computational approaches have been fully developed in other computational biology fields, such as microRNA-disease association prediction. Inspired by the advanced computational methods in these fields, we expect to develop more effective computational model.

\section{Conclusion}

Identifying potential microbe-disease associations is a primary step towards understanding the pathological mechanism of human diseases. In this study, we proposed a Weighted Meta-Graph-based computational method for disease-microbe association prediction. We compared our method with several state-of-the-art methods based on database HMDAD. According to the experimental results, it indicated that our method performed better than baseline methods. Also, we applied our method to make predictions for three common human diseases to validate its effectiveness for new diseases. As a result, our method achieved a desired prediction performance. In addition, in case studies, most of the inferred candidate microbes could be validated by previous reports. Therefore, we believe that the proposed method has potential to investigate the underlying pathological mechanism of human diseases.

\section{Methods}

\section{Human microbe-disease associations}

The known experimentally validated human microbedisease association data were retrieved from Human Microbe-Disease Association Database (HMDAD, http:// www.cuilab.cn/hmdad.) which contains 483 distinct experimentally validated microbe-disease entries involving 39 diseases and 292 microbes [21]. For the sake of convenience, we construct an adjacency matrix $A \in$ $R^{n_{d} \times n_{m}}$ to represent the known human microbe-disease 
associations, where $n_{d}$ denotes the number of diseases while $n_{m}$ the number of microbes. If there exists experimentally confirmed association between disease $d_{i}$ and microbe $m_{j}$, then $A_{i j}$ equals to 1 , otherwise 0 .

\section{Microbe functional similarity}

In this paper, we calculate microbe functional similarity based on the method proposed by Kamneva et al. [61]. In order to accurately calculate the functional similarity for a given pair of microbes, we first need to obtain a proteinprotein functional association network where the nodes represent gene families encoded by either of the genomes and the links represent gene neighbor score values based on STRING database (https://string-db.org.). Gene families are labeled to denote if a protein from a given gene family is present in genome $A$, genome $B$, or both, which produces 3 types of gene families. There exist 6 types of undirected edges (both to $\mathrm{A}$, both to $\mathrm{B}$, both to both, $\mathrm{A}$ to $A, A$ to $B$ and $B$ to $B$ ) in such a network. We define the microbe functional similarity between two microbes as a fraction of edges which cross organismal boundaries (i.e. A to B) among all the edges connecting gene families encoded exclusively in one of the genomes (i.e. A to B, A to $A$ and $B$ to $B$ ). A simple example is shown in Additional file 3: Figure $S 2$. The similarity scores are transformed into a $n_{m} \times n_{m}$ microbe functional similarity matrix $F S$ where $F S\left(m_{i}, m_{j}\right)$ represents the similarity between microbe $m_{i}$ and microbe $m_{j}$.

\section{Disease semantic similarity}

Mesh (Medical Subject Headings) database (http://www. ncbi.nlm.nih.gov/.) includes a plenty of descriptors about diseases, based on which a Directed Acyclic Graph (DAG) can be constructed to describe a disease [62]. The DAG of disease $D$ is composed of not only its ancestor nodes and $D$ itself but also the directed edges from patient nodes to child nodes. Based on the DAG, we can define the contribution value of disease $d$ in $D A G(D)$ to the semantic value of disease $D$ as follows:

$$
\left\{\begin{array}{lc}
S V_{D}(d)=1, & \text { if } d=D, \\
S V_{D}(d)=\max \left\{\Delta * S V_{D}\left(d^{\prime}\right) \mid d^{\prime} \in \text { children of } d\right. & \text { if } d \neq D,
\end{array},\right.
$$

where $\Delta$ represents the semantic contribution decay factor utilized to limit the effects of diseases with different distances to disease $D$ (According to Wang et al. [63], we set $\Delta$ as 0.5 ). Generally, the larger the distance of disease $D$ to its ancestor disease is, the less its contribution to the semantic value of disease $D$ is. The semantic value of disease $D$ can be defined as follows:

$$
S V(D)=\sum_{d \in T(D)} S V_{D}(d)
$$

where $T(D)$ represents all the ancestor diseases of disease $D$ and $D$ itself. Based on the assumption that the larger the shared part of the DAGs of two diseases is, the greater their similar score is, the semantic similarity value between disease $d_{i}$ and disease $d_{j}$ could be defined as follows:

$$
S S\left(d_{i}, d_{j}\right)=\frac{\sum_{t \in T\left(d_{i}\right) \cap T\left(d_{j}\right)}\left(S V_{d_{i}}(t)+S V_{d_{j}}(t)\right)}{S V\left(d_{i}\right)+S V\left(d_{j}\right)} .
$$

\section{Gaussian interaction profile kernel similarity for microbes}

Based on the assumption that microbes with similar functions tend to present interaction or non-interaction with similar diseases [21], we construct microbe similarity network and disease similarity network via known experimentally confirmed human microbe-disease interaction relationships using Gaussian kernel interaction profile, respectively. For a specific microbe $m_{i}$, the corresponding interaction profile could be denoted as $I P\left(m_{i}\right)$, which describes the interaction relationships between microbe $m_{i}$ and all considered diseases, i.e., if a disease is confirmed experimentally to be associated with $m_{i}$, the corresponding value of $I P\left(m_{i}\right)$ equals to 1 , otherwise 0 . According to the interaction profiles, the Gaussian kernel microbe similarity $G M$ can be calculated and defined as follows [29]:

$$
\begin{aligned}
& \operatorname{GM}\left(m_{i}, m_{j}\right)=\exp \left(-\lambda_{m}\left\|I P\left(m_{i}\right)-I P\left(m_{j}\right)\right\|^{2}\right), \\
& \lambda_{m}=\lambda_{m}^{\prime} /\left(\frac{1}{n_{m}} \sum_{i=1}^{n_{m}}\left\|I P\left(m_{i}\right)\right\|^{2}\right),
\end{aligned}
$$

where $\lambda_{m}$ represents the normalized kernel bandwidth, and can be updated by another normalized bandwidth $\lambda_{m}^{\prime}$. For convenience, we set $\lambda_{m}^{\prime}=1$ according to previous relevant research [63]. $n_{m}$ is the number of microbes. $G M(i, j)$ at the $i^{t h}$ row and $j^{\text {th }}$ column denotes the similarity between microbe $m_{i}$ and $m_{j}$.

\section{Gaussian interaction profile kernel similarity for diseases} Similarly, the Gaussian kernel disease similarity $G D$ can be computed as follows:

$$
\begin{aligned}
& G D\left(d_{i}, d_{j}\right)=\exp \left(-\lambda_{d}\left\|I P\left(d_{i}\right)-I P\left(d_{j}\right)\right\|^{2}\right), \\
& \lambda_{d}=\lambda_{d}^{\prime} /\left(\frac{1}{n_{d}} \sum_{i=1}^{n_{d}}\left\|I P\left(d_{i}\right)\right\|^{2}\right),
\end{aligned}
$$

where $\lambda_{d}^{\prime}$ is also set to 1 and $n_{d}$ is the number of diseases. $G D(i, j)$ at the $i^{\text {th }}$ row and $j^{\text {th }}$ column implies the similarity between disease $d_{i}$ and $d_{j}$.

\section{Integrated similarity for diseases}

In order to complement and improve disease similarity, we construct a new similarity network for diseases by 
combining multiple disease similarity networks calculated from different perspective, namely the disease semantic similarity and the Gaussian kernel disease similarity, as is mentioned above. Specifically, the integrated disease similarity can be defined as follows:

$$
D S\left(d_{i}, d_{j}\right)=\left\{\begin{array}{l}
\alpha S S\left(d_{i}, d_{j}\right)+(1-\alpha) G D\left(d_{i}, d_{j}\right), \\
\text { if } d_{i} \text { and } d_{j} \text { has semantic similarity }, \\
G D\left(d_{i}, d_{j}\right), \quad \text { otherwise, }
\end{array}\right.
$$

where $\alpha$ is weight factor defined to limit the effects of the disease semantic similarity and the Gaussian kernel disease similarity to the combined disease similarity. The values of these parameters are determined by the experimental results.

\section{Integrated similarity for microbes}

Similarly, a new similarity network for microbes is constructed by integrating microbe functional similarity and Gaussian kernel microbe similarity. Formally, the integrated microbe similarity can be calculated as follows:

$$
M S=\beta F S+(1-\beta) G M
$$

where $\beta$ is a weight factor used to weight the impacts of the Gaussian kernel microbe similarity and the microbe functional similarity to the final combined microbe similarity.

\section{Construction of heterogeneous information network}

Based on the calculated similarities for diseases and microbes, we can further construct disease similarity network and microbe similarity network, based on which a HIN can be constructed through known experimentally validated MDAs. As for microbe similarity network, $M=\left\{m_{1}, m_{2}, \ldots, m_{n_{m}}\right\}$ implies the node set of microbes and the edge weights denote the similarities between microbes. Similarly, as for the disease network, $D=$ $\left\{d_{1}, d_{2}, \ldots, d_{n_{d}}\right\}$ denotes the node set of diseases and the edge weights represent the similarities between diseases. In addition, a bipartite network is also constructed in the HIN with the node set consisting of microbe and disease nodes and the edge weights representing the absence or presence of relationships between diseases and microbes, i.e., if there is an edge between $d_{i}$ and $m_{j}$, it implies that $d_{i}$ is experimentally confirmed to be related with $m_{j}$ and the corresponding edge weight equals to 1 , otherwise 0 .

\section{WMGHMDA}

In this work, we developed a novel Weighted Meta-Graph based computational framework for predicting microbedisease associations (WMGHMDA). The flowchart of WMGHMDA is shown in Fig. 5, Firstly, to improve the completeness of similarity, we obtain the integrated disease similarity by combining disease semantic similarity with Gaussian kernel disease similarity, and the integrated microbe similarity by combining microbe functional similarity with Gaussian kernel microbe similarity, based on which a HIN is constructed via known microbe-disease interaction network. Secondly, we design a Weighted Meta-Graph search algorithm and implement it on the HIN to calculate the probability score for each microbedisease pair. Finally, for a disease, all candidate microbes are prioritized according to their probability scores.

\section{Meta-graph}

The concept of meta-graph has been developed to capture more complex semantics in HIN that meta-path cannot[64]. Since each particular meta-graph represents an essential semantic unit between a source node and a target node in HIN, meta-graph has been widely applied in representation learning and recommendation system [65-68]. Inspired by this, we extend meta-graph to solve the problem of uncovering missing MDAs based on HIN. Here, we focus on the concepts related to our paper. Specially, we define the meta-graph in heterogeneous biological network for prediction.

Meta-graph is the subset of HIN schema. Formally, meta-graph could be defined as sub-graph $G_{s}=(V, E)$, where $V=\left\{d_{i} \mid i=1,2, \ldots, n_{d}\right\} \cup\left\{m_{j} \mid j=1,2, \ldots, n_{m}\right\}$ represents the set of nodes including diseases and microbes, and $E=\left\{\left(v_{i}, v_{j}\right) \mid i, j=1,2, \ldots, n, n \in\left(n_{d} \cup n_{m}\right)\right\}$ implies the set of edges including inter-layer relationship connections in the bipartite network and intra-layer similarity connections in both of disease similarity network and microbe similarity network. A meta-path is a special case of a meta-graph. Here, we call it meta-graph uniformly. Figure 6 displays six types of meta-graphs which depict possible semantic relations between a seed disease node and a target microbe node. Here we regard the products of the weight values of all edges existing in a meta-graph as its contribution value to the prediction probability of the microbe-disease association pair. For example, for the given disease $d_{i}$ and microbe $m_{j}$, the contribution value of a meta-graph to the probability score of the pair could be defined and calculated as follows if there exists no observed relationship between them (assuming that the meta-graph is linear and includes less than three intermediate nodes):

$$
P\left(d_{i}, m_{j}\right)=\sum_{k=1}^{n_{d}} \sum_{t=1}^{n_{m}} D S\left(d_{i}, d_{k}\right) A\left(d_{k}, m_{t}\right) M S\left(m_{t}, m_{j}\right) .
$$

\section{Weighted meta-graph based prediction model}

In order to more accurately capture potential subtle semantics between disease nodes and microbe nodes, 


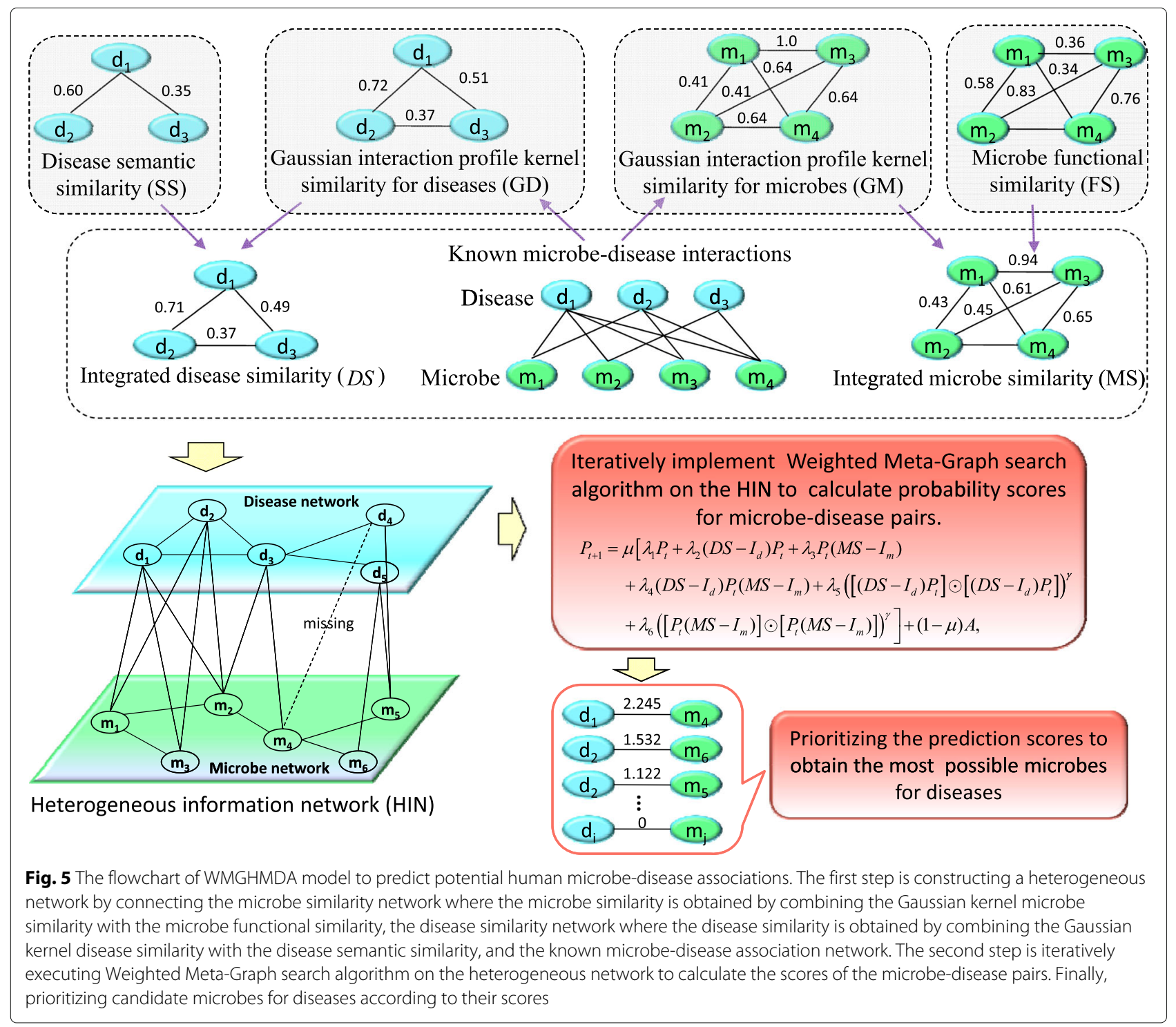

here we generalize common unweighted meta-graph to weighted meta-graph. In the weighted meta-graph, the weight values of intra-layer edges represent the similarities between diseases or microbes, and the weight values of inter-layer edges denote the possibilities of existing associations between diseases and microbes, i.e., if a disease is experimentally verified to be related to a microbe, the weight value of corresponding bipartite edge equals to 1 , otherwise 0 . Empirically, as the number of the edges and the intermediate nodes of a meta-graph increases, the importance of the meta-graph also gradually decreases. Thus, based on this, we only adopt six types of weighted meta-graph patterns (as shown in Fig. 6), with the number of edges less than five or the number of intermediate nodes less than three, to identify latent MDAs in HIN. They include weighted meta-graphs with singlepath (such as Fig. 6a,b,c,d) and weighted meta-graphs with dual-path (such as Fig. 6e,f). With the above-mentioned definition of the contribution of meta-graph, for these six different types of weighted meta-graphs, the corresponding contribution values could be described as follows according to formula (10), respectively:

$$
P_{a}\left(d_{i}, m_{j}\right)=A\left(d_{i}, m_{j}\right)
$$

$$
\begin{aligned}
& P_{b}\left(d_{i}, m_{j}\right)=\sum_{k=1, k \neq i}^{n_{d}} D S\left(d_{i}, d_{k}\right) A\left(d_{k}, m_{j}\right), \\
& P_{c}\left(d_{i}, m_{j}\right)=\sum_{t=1, t \neq j}^{n_{m}} A\left(d_{i}, m_{t}\right) M S\left(m_{t}, m_{j}\right),
\end{aligned}
$$




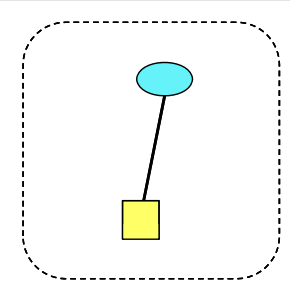

(a)

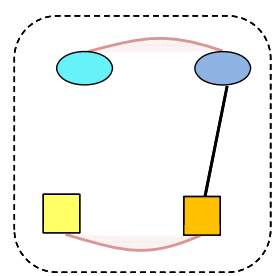

(d)

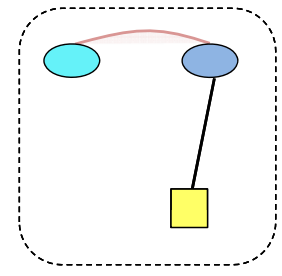

(b)

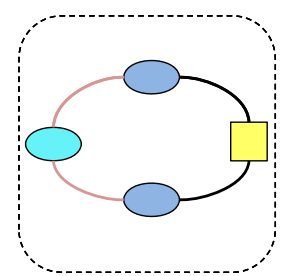

(e)

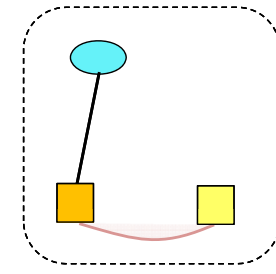

(c)

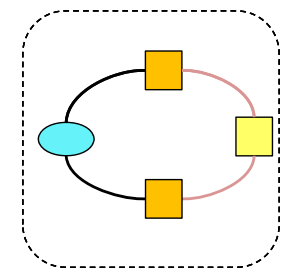

(f)

Fig. 6 Examples of weighted meta-graphs used for microbe-disease association prediction. Red line represents the similarity linking between diseases that weights similarity degree; Black line is bipartite linking that denotes whether a disease is associated with a microbe or not, i.e., if a disease is confirmed to be related to a microbe, the weight value of corresponding edge equals to 1 , otherwise 0 . The numbers of the given nodes directly connected to the bipartite edge are $2,1,1,0,1,1$ from $(\mathbf{a})$ to $(\mathbf{f})$, respectively

$$
\begin{aligned}
P_{d}\left(d_{i}, m_{j}\right)= & \sum_{k=1, k \neq i}^{n_{d}} \sum_{t=1, t \neq j}^{n_{m}} \\
& {\left[D S\left(d_{i}, d_{k}\right) A\left(d_{k}, m_{t}\right) M S\left(m_{t}, m_{j}\right)\right], }
\end{aligned}
$$

$$
\begin{aligned}
P_{e}\left(d_{i}, m_{j}\right)= & \sum_{k=1, k \neq i}^{n_{d}} \sum_{t=1, t \neq i, t \neq k}^{n_{d}} \\
& {\left[D S\left(d_{i}, d_{k}\right) A\left(d_{k}, m_{j}\right) D S\left(d_{i}, d_{t}\right) A\left(d_{t}, m_{j}\right)\right], }
\end{aligned}
$$

$$
\begin{aligned}
P_{f}\left(d_{i}, m_{j}\right)= & \sum_{k=1, k \neq j}^{n_{m}} \sum_{t=1, t \neq j, t \neq k}^{n_{m}} \\
& {\left[A\left(d_{i}, m_{k}\right) M S\left(m_{k}, m_{j}\right) A\left(d_{i}, m_{t}\right) M S\left(m_{t}, m_{j}\right)\right] . }
\end{aligned}
$$

However, weighted meta-graphs with different structure characteristics could actually yield bias contributions to a microbe-disease pair. Here, for identifying this bias, we introduce bias rating to describe the differentiated contributions of different weighted meta-graphs. The main differences between weighted meta-graphs depend on the number of the given nodes. Here, the given node refers to the node that is directly connected to the bipartite edge and could be a seed disease node as well as a target microbe node. Specifically, as shown in Fig. 6, different numbers of the given nodes are included in these six kinds of weighted meta-graphes. For example, the numbers of the given nodes for Fig. 6a-f are 2, 1, 1, 0, 1, 1, respectively. Based on the assumption that meta-graph with more given nodes has greater contribution potential, it indicates that compared with the other weighted metagraphs, Fig. 6a possibly has more potential to contribute useful information to an association pair while Fig. $6 \mathrm{~d}$ could contribute the least useful information. Note that although Fig. $6 \mathrm{~b}, \mathrm{c}$ have the same numbers of the given nodes as Fig. 6e,f, the later may play more important role in predicting candidate microbes for diseases. It can be explained that both Fig. 6e and f are dural-path weighted meta-graphs, which implies that a seed disease node has more semantic paths simultaneously connecting it to a target microbe node in such meta-graph. In other words, such weighted meta-graph can hide more prolific semantic information, implying more contribution potential. Therefore, depending on the potential of contribution, we assign different bias ratings for different weighted metagraphs. The greater the potential of contribution is, the higher the bias rating is.

According to the definition of weighted meta-graph, it can be found that a given microbe-disease association pair can be hidden in multiple varieties of weighted meta-graphs in HIN. Based on the assumption that more weighted meta-graphs are determined to be related to a microbe-disease pair, the pair is more likely to have association, the accumulating contribution values of all weighted meta-graphs connecting a seed disease with a target microbe can be served as their final prediction probability. Mathematically, for specific disease $d_{i}$ and microbe $m_{j}$, after implementing Weighted Meta-Graph 
search algorithm on the HIN to traverse all relevant weighted meta-graphs, the prediction score $\bar{P}$ could be defined and calculated by summing up the contribution values of these weighted meta-graphs as follows:

$$
\bar{P}\left(d_{i}, m_{j}\right)=\sum_{l=1}^{N} \sum_{r=1}^{M} \lambda_{l}^{r} P_{l}^{r}\left(d_{i}, m_{j}\right)
$$

where $P_{l}^{r}\left(d_{i}, m_{j}\right)$ denotes the contribution value of the $r^{\text {th }}$ meta-graph belonging to the $l^{\text {th }}$ type of weighted metagraph to the pair $\left(d_{i}, m_{j}\right), N(N=6)$ represents the category number of weighted meta-graph, and $M$ denotes the number of the weighted meta-graph included in a specific weighted meta-graph pattern. $\lambda(\lambda \in[0,1])$ is bias rating applied to distinguish the contributions of different types of weighted meta-graphs to the final predicted probability $\bar{P}$. It is noteworthy that all weighted meta-graphs in the same category are considered to present identical bias ratings on a microbe-disease pair. We iteratively implement the above search progress based on Weighted Meta-Graph search algorithm until the prediction probability matrix $P_{t}$ converges and describe the iteration formula with matrix formation as follows:

$$
\begin{aligned}
P_{t+1}= & \mu\left[\lambda_{1} P_{t}+\lambda_{2}\left(D S-I_{d}\right) P_{t}+\lambda_{3} P_{t}\left(M S-I_{m}\right)\right. \\
& +\lambda_{4}\left(D S-I_{d}\right) P_{t}\left(M S-I_{m}\right) \\
& +\lambda_{5}\left(\left[\left(D S-I_{d}\right) P_{t}\right] \odot\left[\left(D S-I_{d}\right) P_{t}\right]\right)^{\gamma} \\
& \left.+\lambda_{6}\left(\left[P_{t}\left(M S-I_{m}\right)\right] \odot\left[P_{t}\left(M S-I_{m}\right)\right]\right)^{\gamma}\right] \\
& +(1-\mu) A,
\end{aligned}
$$

where $I_{d}$ and $I_{m}$ represent unit matrices with the sizes of $n_{d}$ and $n_{m}$, respectively, and $\lambda$ is bias rating (According to the experimental results, the best performance is obtained when $\lambda_{1}=0.35, \lambda_{2}=0.1, \lambda_{3}=0.1, \lambda_{4}=0.05, \lambda_{5}=$ 0.2 and $\lambda_{6}=0.2$.). The element of probability matrix $P_{t}$ at the $i^{\text {th }}$ row and $j^{\text {th }}$ column means the probability score of association between disease $d_{i}$ and microbe $m_{j}$ at step $k . \odot$ denotes Hadamard product, $\gamma$ is a decay coefficient used to control the contributions of dural-path weighted metagraphs (Here, we set $\gamma$ as 0.1 .), and $\mu \in(0,1)$ is a decay factor similar to the restart probability in the random walk with restart. The initial values of probability matrix $P_{t}$ is defined as the normalized adjacent matrix $A$. According to Wang et al. [69], it assures that formula (18) will converge if $D S$ and $M S$ are properly normalized using Eqs. (19) and (20), respectively.

$$
\begin{aligned}
& D S\left(d_{i}, d_{j}\right)=\frac{D S\left(d_{i}, d_{j}\right)}{\sqrt{\sum_{l=1}^{n_{d}} D S\left(d_{i}, d_{l}\right)} \cdot \sqrt{\sum_{l=1}^{n_{d}} D S\left(d_{l}, d_{j}\right)}}, \\
& M S\left(m_{i}, m_{j}\right)=\frac{M S\left(m_{i}, m_{j}\right)}{\sqrt{\sum_{l=1}^{n_{m}} M S\left(m_{i}, m_{l}\right)} \cdot \sqrt{\sum_{l=1}^{n_{m}} M S\left(m_{l}, m_{j}\right)}} .
\end{aligned}
$$

After some steps, the prediction probability $P_{t}$ is steady, according to which all candidate microbes for each disease could be prioritized. The top microbes are considered as the most possible microbes associated with the given disease.

The main time complexity of the algorithm is from the search of meta-graph and the corresponding calculation of contribution values. Given that the numbers of disease and microbe are $n_{d}$ and $n_{m}$, respectively, for six types of weighted meta-graphs (i.e. Fig. 6a-f), this process takes $O\left(n_{d} n_{m}\right), O\left(n_{d}^{2} n_{m}\right), O\left(n_{d} n_{m}^{2}\right), O\left(n_{d}^{2} n_{m}^{2}\right), O\left(n_{d}^{3} n_{m}\right)$ and $O\left(n_{d} n_{m}{ }^{3}\right)$ in the worst case scenario by considering each disease node as seed node while each microbe node as target node, respectively. Therefore, the time complexity of the algorithm is $O\left(n_{d} n_{m}\right.$ $\left.\left(n_{d}+n_{m}\right)^{2}\right)$. Our algorithm is implemented on Matlab R2016a.

\section{Implement wMGHMDA on new diseases}

For new diseases which lack known associated microbes in the database but have other features available for prediction, few previous computational methods could be applied to make predictions. We implement WMGHMDA on new diseases for exploring potential microbes. One of the advantages of weighted meta-graph is that it is able to effectively capture the hidden semantic associations for microbe-disease pairs on the HIN. WMGHMDA embeds weighted meta-graph with multiple prior features related to diseases and microbes, such as disease semantic similarity and microbe functional similarity, which provides a possibility to bridge a new disease node with microbe node in HIN. Therefore, for new diseases, although there are no evidences to confirm their associations between microbes and them, WMGHMDA can still be applied to make predictions. An example is shown in Fig. 7.

Given a specific unlabelled disease $d_{3}$, for each of association pairs between $d_{3}$ and $m_{j}$, it is easy to seek relevant weighted meta-graphs hidden in the HIN. For example, we can find two types of weighted meta-graphs related to association pair $d_{3}-m_{4}$, such as weighted metagraphs consisting of $d_{3}, d_{4}, m_{4}$ and $d_{3}, d_{2}, m_{2}, m_{4}$, respectively. If there is a higher similarity between the node $d_{3}$ and a labeled disease node $d_{i}$ (i.e., $d_{2}$ ) or between the specific microbe node $m_{j}$ (i.e., $m_{4}$ ) and a labeled microbe node $m_{k}$ (i.e., $m_{2}$ ), it means that disease $d_{3}$ is associated with microbe $m_{j}$ with greater probability. After the Weighted Meta-Graph search algorithm is implemented, each microbe in the HIN will obtain a probability score denoting the possibility of being associated with new disease $d_{3}$. The greater score indicates closer interaction between the microbes and $d_{3}$. The probability scores can be calculated according to formula (17). 


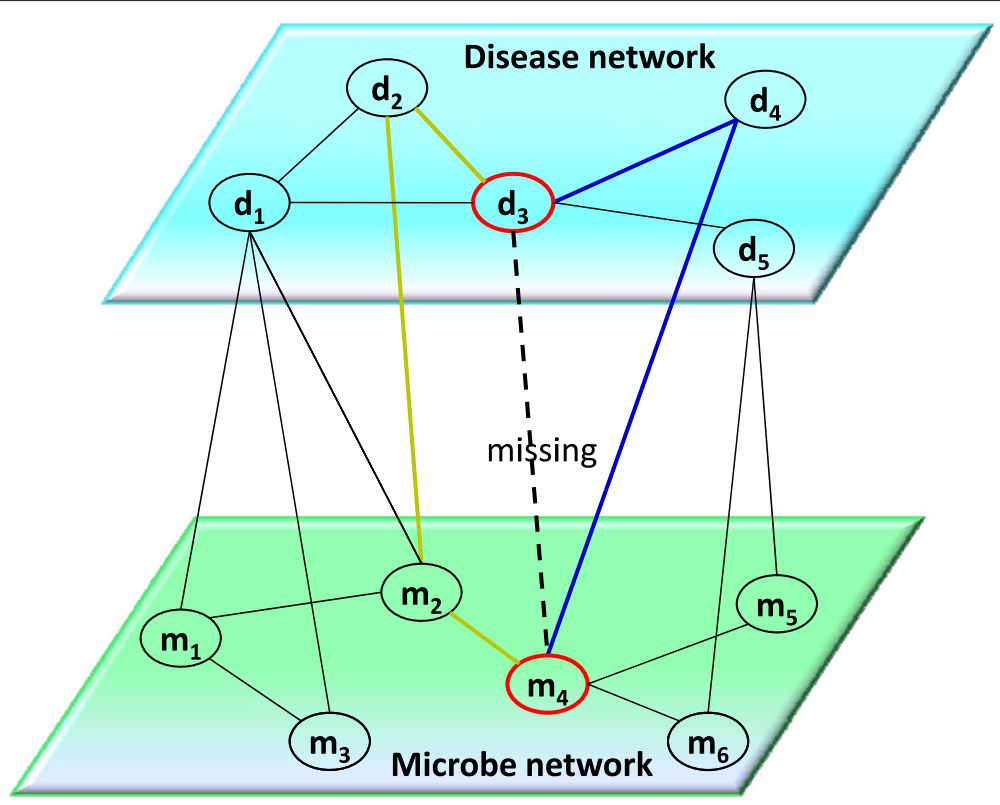

Fig. 7 The heterogeneous information network. Two types of weighted meta-graphs (i.e. Fig. 6b,d) can be utilized to obtain the probability score of interaction between new disease node $d_{3}$ and candidate microbe node $m_{4}$, such as weighted meta-graphs consisting of $d_{3}, d_{4}, m_{4}$ and $d_{3}, d_{2}, m_{2}, m_{4}$, respectively. $d_{3}$ represents unlabeled disease; $d_{1}, d_{2}, d_{4}$ and $d_{5}$ denote labeled disease; $m_{1}, m_{2}, m_{3}, m_{4}, m_{5}$ and $m_{6}$ represent labeled microbes; Solid lines and dotted line in the bipartite network means known and unknown bipartite linkings, respectively

\section{Supplementary information}

\section{Supplementary information accompanies this paper at}

https://doi.org/10.1186/s12859-019-3066-0.

Additional file 1: Table S1. Performance comparisons between our method and baseline methods (NTSHMDA, nGRHMDA, biRWHMDA, IRLSHMDA, KATZHMDA, bDSILP and pRWHMDA) in terms of precision, recall, and f1-score, respectively.

Additional file 2: Figure S1. Network of the top-50 predicted associations for iBD and asthma obtained by our method. ellipses with Orange and circles represent diseases and microbes, respectively. the blue lines and red lines denote the associations of predicted microbes with iBD and asthma, respectively.

Additional file 3: Figure S2. A simple example of how microbe functional similarity is calculated.

\section{Abbreviations}

LOOCV: Leave-one-out cross validation; AUC: Area under ROC curve; DAG: Directed acyclic graph; HIN: Heterogeneous information network; HMDAD: Human microbe-disease association database; RWR: Random walk with restart; MDAs: Microbe-disease associations; WMGHMDA: Weighted meta-graph-based method for human microbe disease association prediction

\section{Acknowledgements}

Not applicable.

\section{Authors' contributions}

YHL and JWL initiated the study. YHL developed the code, conducted the experiments and wrote the manuscript. All authors read and approved the final version.

\section{Funding}

This work was supported by the National Natural Science Foundation of China (Grant no. 61873089, 61572180). The funding bodies did not play any role in the design of the study and collection, analysis, and interpretation of data and in writing the manuscript.

\section{Availability of data and materials}

The datasets supporting the conclusions of this article are included within the article and its Additional files. The code used in the current study is available at https://github.com/yahuilong/WMGHMDA.

\section{Ethics approval and consent to participate}

Not applicable.

\section{Consent for publication}

Not applicable.

\section{Competing interests}

The authors declare that they have no competing interests.

Received: 4 July 2019 Accepted: 2 September 2019

Published online: 01 November 2019

\section{References}

1. Holmes E, Wijeyesekera A, Taylorrobinson SD, Nicholson J-K. The promise of metabolic phenotyping in gastroenterology and hepatology. Nat Rev Gastroenterol Hepat. 2015;12(8):458-71.

2. Ventura M, OFlaherty S, Claesson MJ, Francesca T, Todd RK, Douwe VS, Paul WO. Genomescale analyses of health-promoting bacteria: probiogenomics. Nat Rev Microbiol. 2009;7:61-71.

3. Sommer F, Backhed F. The gut microbiota-masters of host development and physiology. Nat Rev Microbiol. 2013;11:227-38.

4. Consortium HMP. A framework for human microbiome research. Nature. 2012:486(7402):215-21.

5. Consortium HMP. Structure, function and diversity of the healthy human microbiome. Nature. 2012;486(7402):207-14.

6. Gill SR, Pop M, DeBoy RT, Eckburg PB, Turnbaugh PJ, Samuel BS, GORDON JI, Relman DA. Metagenomic analysis of the human distal gut microbiome. Science. 2006;312(5778):1355-9.

7. Khachatryan ZA, Ktsoyan Z, Manukyan G, Denise K, Ghazaryan KA, Aminov R. Predominant role of host genetics in controlling the composition of gut microbiota. PLoS ONE. 2008;3(8):e3064 
8. Turnbaugh PJ, Hamady M, Yatsunenko T, Cantarel BL, Duncan A, Ley RE, Sogin ML, Jones WJ, Roe BA, Affourtit JP, Egholm M, Henrissat B, Heath AC, Knight R, Gordon Jl. A core gut microbiome in obese and lean twins. Nature. 2009;457(7228):480-4.

9. Goodrich JK, Waters JL, Poole AC, Sutter JL, Koren O, Blekhman R, Beaumont M, Treuren WV, Knight R, Bell JT, Spector TD, Clark AG, Ley RE. Human genetics shape the gut microbiome. Cell. 2014;159(4):789-99.

10. Davenport ER, Man OM, Michelini K, Barreiro LB, Ober C, Gilad Y. Seasonal variation in human gut microbiome composition. PLoS ONE. 2014;9(3):e90731.

11. Mason MR, Preshaw PM, Nagaraja HN, Dabdoub SM, Rahman A, Kumar PS. The subgingival microbiome of clinically healthy current and never smokers. ISME J. 2015;9:268-72.

12. David LA, Maurice CF, Carmody RN, Gootenberg DB, Button JE, Wolfe BE, Ling AV, Devlin AS, Varma Y, Fischbach MA4, Biddinger SB, Dutton RJ, Turnbaugh PJ. Diet rapidly and reproducibly alters the human gut microbiome. Nature. 2014;505(7484):559-63.

13. Donia MS, Cimermancic P, Schulze CJ, Wieland Brown LC, Martin J, Mitreva M, Clardy J, Linington RG, Fischbach MA. A systematic analysis of biosynthetic gene clusters in the human microbiome reveals a common family of antibiotics. Cell. 2014;158(6):1402-14.

14. Rivas MN, Crother TR, Arditi M. The microbiome in asthma. Curr Opin Pediatr. 2016;28(6):764-71.

15. Wen L, Ley RE, Volchkov PY, Stranges PB, Avanesyan L, Stonebraker AC, Hu C, Wong FS, Szot GL, Bluestone JA, Gordon Jl, Chervonsky AV. Innate immunity and intestinal microbiota in the development of type 1 diabetes. Nature. 2008;455(7216):1109-13.

16. Henao-Mejia J, Elinav E, Thaiss CA, Licona-Limon P, Flavell RA. Role of the intestinal microbiome in liver disease. J Autoimmun. 2013;46:66-73.

17. Schwabe RF, Jobin C. The microbiome and cancer. Nat Rev Cancer. 2013;13(11):800-12.

18. Huang YJ, Nelson CE, Brodie EL, Desantis TZ, Baek MS, Liu J, Woyke T, Allgaier M, Bristow J, Wiener-Kronish JP, Sutherland ER, King TS, Icitovic N, Martin RJ, Calhoun WJ, Castro M, Denlinger LC, Dimango E, Kraft M, Peters SP, Wasserman SI, Wechsler ME, Boushey HA, Lynch SV. Airway microbiota bronchial hyperresponsiveness in patients with suboptimally controlled asthma. J Allergy Clin Immunol. 2011;127(2):372-81.

19. Larsen N, Vogensen FK, Berg FWJ, Nielsen DS, Aadreasen AS, Pedersen BK, Soud WAA, Sorense SJ, Hansen LH, Jakobsen M. Gut microbiota in human adults with type 2 diabetes differs from non-diabetic adults. PLoS ONE. 2010;5(2):e9085.

20. Moore WE, Moore LH. Intestinal floras of populations that have a high risk of colon cancer. Appl Environ Microbiol. 1995;61(9):3202-7.

21. Ma W, Zhang L, Zeng P, Huang C, Li J, Geng B, Yang J, Kong W, Zhou $X$, Cui $Q$. An anlaysis of human microbe-disease associations. Brief Bioinforma. 2017;18(1):85-97.

22. Chen X, Huang YA, You ZH, Yan GY, Wang XS. A novel approach based on KATZ measure to predict associations of human microbiaota with non-infectious diseases. Bioinformatics. 2017;33(5):733-9.

23. Shen $X J$, Chen $Y$, Jiang $X P, H u X H, H e T T$, Yang JC. Predicting disease microbe association by random walking on the heterogeneous network. In: IEEE International Conference on Bioinformatics and Biomedicine; 2016. https://doi.org/10.1109/BIBM.2016.7822619.

24. Luo JW, Long YH. NTSHMDA: Prediction of Human Microbe-Disease Association based on Random Walk by Integrating Network Topological Similarity. IEEE/ACM Trans Comput Biol Bioinforma. 2018. https://doi.org/ 10.1109/TCBB.2018.2883041.

25. Huang ZA, Chen X, Zhu Z, Liu H, Yan GY, You ZH, Wen Z. PBHMDA: Path-based human microbe-disease association prediction. Front Microbiol. 2017;8:233.

26. Huang YA, You ZH, Chen X, Huang ZA, Zhang SW, Yan GY. Prediction of microbe disease association from the integration of neighbor and graph with collaborative recommendation model. J Transl Med. 2017;15:209.

27. Zhang W, Yang WT, Lu XT, Huang F, Luo F. The Bi-direction similarity integration method for predicting microbe-disease association. IEEE Access. 2017:99:1

28. Liu Y, Luo JW, Ding PJ. Inferring MicroRNA Targets based on Restricted Boltzman Machines. IEEE J Biomed Health Inform. 2017;23(1):427-36.

29. Chen X, Yan GY. Novel human LncRNA-disease association inference based on IncRNA expression profiles. Bioinformatics. 2013;29(20):2617-24.
30. Chen X, Yan CC, Zhang X, You ZH. Long non-coding RNAs and complex diseases: from experimental results to computational models. Brief Bioinforma. 2016;18(4):558-76.

31. Chen X, Ren B, Chen M, Wang QX, Zhang LX, Yan GY. NLLSS: Predicting synergistic drug combinations based on semi-supervised learning. PLoS Comput Biol. 2016;12(7):e1004975.

32. Chen X, Yin J, Qu J, Huang L. MDHGI: Matrix decomposition and heterogeneous graph inference for miRNA-disease association prediction. PLoS Comput Biol. 2018;14(8):e1006418.

33. Xiao Q, Luo J, Liang C, Cai J, Ding P. A graph regularized non-negative matrix factorization method for identifying microRNA-disease associations. Bioinformatics. 2018;34(2):239-48.

34. Chen X, Xie D, Zhao Q, You ZH. MicroRNAs and complex diseases: from experimental results to computational models. Brief Bioinforma. 2019;20(2):515-39.

35. Li Y, Liang C, Wong KC, Luo JW, Zhang ZL. Mirsynergy: detecting synergistic miRNA regulatory modules by overlapping neighbourhood expansion. Bioinformatics. 2014;30(18):2627-35.

36. Wang F, Huang ZA, Chen X, Zhu Z, Wen Z, Zhao J, Yan GY. LRLSHMDA: Laplacian regularized least squares for human microbe-disease association prediction. Sci Rep. 2017;7:7601.

37. He BS, Peng LH, Li ZJ. Human microbe-disease association prediction with graph regularized non-negative matrix factorization. Front Microbiol. 2018. https://doi.org/10.3389/fmicb.2018.02560.

38. Shen Z, Jiang ZC, Bao WZ. CMFHMDA: Collaborative matrix factorization for human microbe-disease association prediction. Intell Comput Theor Appl. 2017261-9. https://doi.org/10.1007/978-3-319-63312-1_24.

39. Bao WZ, Jiang ZC, Huang DS. Novel human microbe-disease association prediction using network consistency projection. BMC Bioinformatics. 2017;18 Suppl 16:543.

40. Wang L, Ping PY, Kuang LN, Ye ST, Buland LFM, Pei TR. A novel approach based on bipartite network to predict human microbe-disease associations. Curr Bioinforma. 2018;13(2):141-8.

41. Zou S, Zhang JP, Zhang ZP. A novel approach for predicting microbe-disease associations by bi-random walk on the heterogeneous network. PLoS ONE. 2017;12(9):e0184394.

42. Wu CY, Gao R, Zhang DL, Han SY, Zhang Y. PRWHMDA: Human microbe-disease association prediction by random walk on the heterogeneous networks with PSO. Int J Biol Sci. 2018;14(8):849-57.

43. Davis J, Goadrich $M$. The relationship between precision-recall and roc curves. In: 29th International Conference on Machine Learning; 2006. p. 233-40. https://doi.org/10.1145/1143844.1143874.

44. Mordelet F, Vert JP. ProDiGe: Prioritization Of Disease Genes with multitask machine learning from positive and unlabeled examples. BMC Bioinformatics. 2011;12:389.

45. Natarajan N, Dhillon IS. Inductive matrix completion for predicting gene-disease associations. Bioinformatics. 2014;30(13):60-68.

46. Shi HB, Xu J, Zhang GG, Xu LD, Li CQ, Wang L, Zhao Z, Jiang W, Guo Z, $\mathrm{LiX}$. Walking the interactome to identify human miRNA-disease associations through the functional link between miRNA targets and disease genes. BMC Syst Biol. 2013;7:101.

47. Chen X, Wang L, Qu J, Guan NN, Li JQ. Predicting miRNA-disease association based on inductive matrix completion. Bioinformatics. 2018;34(24):4256-65.

48. Fein BT. Bronchial asthma caused by Pseudomonas aeruginosa diagnosed by bronchoscopic examination. Annals Allergy. 1955;13(6):639-41.

49. Marri PR, Stern DA, Wright AL, Billheimer D, Martinez FD. Asthma-associated differences in microbial composition of induced sputum. J Allergy Clin Immunol. 2013;131(2):346-52.

50. Jung JW, Choi JC, Shin JW, Kim JY, Park IW, Choi BW, Park HW, Cho SH, Kim K, Kang HR. Lung microbiome analysis in Steroid-Naive asthma patients by using while sputum. Tuberc Respir Dis. 2016;79(3):165.

51. Li N, Qiu R, Yang Z, Li J, Chung KF, Zhong N, Zhang Q. Sputum mcirobiota in severe asthma patients: Relationship to eosinophilic inflammation. Respir Med. 2017;131:192-8.

52. Yu J, Jang SO, Kim BJ, Song YH, Kwon JW, Kang MJ, Choi WA, Jung HD, Hong SJ. The effects of Lactobacillus rhamnosus on the Prevention of Asthma in a Murine Model. Allergy Asthma Immunol Res. 2010;2(3): 199-205. 
53. Boutin S, Depner M, Stahl M, Graeber SY, Dittrich SA, Legatzki A, von Mutius E, Mall M, Dalpke AH. Comparison of Oropharyngeal Microbiota from Children with Asthma and Cystic Fibrosis. Mediat Inflamm. 20171-10. https://doi.org/10.1155/2017/5047403.

54. Vael C, Nelen V, Verhulst SL, Goossens H, Desager K, Early intestinal bacteroides fragilis colonization developmentofasthma. BMC Pulm Med. 2008;08:19.

55. Thanh HD, Kim SA, Park HK, Shin JW, Park SG, Kim WY. Analysis of Oropharyngeal microbiota between the patients with Bronchial Asthma and the Non-Asthmatic persons. J Bacteriol Virol. 2013;43(4):270.

56. Walters AW, Xu Z, Knight R. Meta-analyses of human gut microbes associated with obesity and IBD. Febs Lett. 2014;588(22):4223-33.

57. Sokol H, Seksik P, Furet JP, Firmesse O, Nion-Larmurier I, Beaugerie L, Cosnes J, Corthier G, Marteau P, Doré J. Low counts of Faecalibacterium prausnitzii in colitis microbiota. Inflamm Bowel Dis. 2009;15(8):1183-9.

58. Sonnenberg A, Genta RM. Low prevalence of Helicobacter pylori infection among patients with inflammatory bowel disease. Aliment Pharmacol Ther. 2012;35(4):469-76.

59. Azimirad M, Bahreiny R, Hasani Z. Prevalence of superantigenic Staphylococcus aureus and toxigenic Clostridium difficile in patients with IBD. In: Conferences Portal University of Medical Sciences; 2012. http:// congress.arums.ac.ir/index.php/IICM/5/paper/view/1246.

60. Said HS, Suda W, Nakagome S, Chinen H, Oshima K, Kim S, Kimura R, Iraha A, Ishida H, Fujita J, Mano S, Morita H, Dohi T, Oota H, Hattori M. Dysbiosis of salivary microbiota in inflammatory bowel disease and its association with oral immunological biomarkers. DNA Res. 2014;21(1): $15-25$.

61. Kamneva OK. Genome composition and phylogeny of microbes predict their co-occurrence in the environment. PLoS Comput. Biol. 2017;13(2): e1005366.

62. Lipscomb CE. Medical subject headings (MeSH). Bull Med Libr Assoc. 2000;88(03):265-6.

63. Wang D, Wang J, Lu M, Song F, Cui Q. Inferring the human microRNA functional similarity and functional network based on microRNA-associated diseases. Bioinformatics. 2010;26(13):1644-50.

64. Sun YZ, Han JW. Mining heterogeneous information networks: principles and methodologies. Synth Lect Data Min Knowl Discov. 2012;3(2):1-159.

65. Dong Y, Chawla NV, Swami A. metapath2vec: Scalable representation learning for heterogeneous networks. In: Proceedings of the 23rd ACM SIGKDD International Conference on Knowledge Discovery and Data Mining; 2017. p. 135-44.

66. Zhao H, Yao QM, Li JD, Song YQ, Lee DL. Meta-Graph based recommendation fusion over heterogeneous information networks. In: Proceedings of the 23rd ACM SIGKDD International Conference on Knowledge Discovery and Data Mining; 2017. p. 635-44. https://doi.org/ 10.1145/3097983.3098063.

67. Fu TY, Lee WC, Lei Z. Hin2vec: Explore meta-paths in heterogeneous information networks for representation learning. In: International Conference on Information and Knowledge Management; 2017. p. 1797-806. https://doi.org/10.1145/3132847.3132953.

68. Shi Y, Gui H, Zhu Q, Kaplan L, Han JW. Aspem: Embedding learning by aspects in heterogeneous information networks. In: International Conference on Data Ming; 2018. arXiv preprint arXiv:1803.01848.

69. Wang WH, Yang S, Li J. Drug target predictions based on heterogeneous graph inference. In: Proceedings of the Pacific Symposium; 2013. p. 53-64. https://doi.org/10.1142/9789814447973_0006.

\section{Publisher's Note}

Springer Nature remains neutral with regard to jurisdictional claims in published maps and institutional affiliations.

Ready to submit your research? Choose BMC and benefit from:

- fast, convenient online submission

- thorough peer review by experienced researchers in your field

- rapid publication on acceptance

- support for research data, including large and complex data types

- gold Open Access which fosters wider collaboration and increased citations

- maximum visibility for your research: over $100 \mathrm{M}$ website views per year

At $\mathrm{BMC}$, research is always in progress.

Learn more biomedcentral.com/submissions 